\title{
Plasma potassium-lowering effect of oral glucose, sodium bicarbonate, and the combination thereof in healthy neonatal dairy calves
}

\author{
W. Grünberg, ${ }^{* 1}$ H. Hartmann, † O. Burfeind,‡ W. Heuwieser,‡ and R. Staufenbiel§ \\ *Department of Farm Animal Health, Universiteit Utrecht, 3584 CL Utrecht, the Netherlands \\ †Institut für Veterinär-Physiologie, \\ †Tierklinik für Fortpflanzung, and \\ §Klinik für Klauentiere, Freie Universität Berlin, 14163 Berlin, Germany
}

\begin{abstract}
Hyperkalemia is a common complication in neonatal diarrheic dairy calves and is corrected by administration of glucose or sodium bicarbonate. Although the hypokalemic effect of glucose is well established in other species, controversial results are reported for sodium bicarbonate. Our objective was to study the effect and mechanism of action of glucose and sodium bicarbonate on the potassium homeostasis of healthy neonatal dairy calves. Nine healthy neonatal HolsteinFriesian calves underwent 3 oral treatments with 2 $\mathrm{L}$ of $\mathrm{NaHCO}_{3}(150 \mathrm{mmol} / \mathrm{L})$, glucose $(300 \mathrm{mmol} / \mathrm{L})$, and glucose $+\mathrm{NaHCO}_{3}$ solution $(300 \mathrm{mmol} / \mathrm{L}+150$ $\mathrm{mmol} / \mathrm{L}$ ) in randomized order. Blood was obtained before treatment $\left(\mathrm{T}_{0}\right)$ and at 30-min intervals thereafter. Changes between each time point and $\mathrm{T}_{0}$ were determined for all parameters. Urine was collected volumetrically to determine total renal potassium excretion over an 8-h posttreatment period. Plasma volume changes were extrapolated from changes in plasma protein concentration. Treatment and time effects were tested with repeated-measures ANOVA. Multivariate stepwise regression analysis using dummy variable coding was conducted to identify associations between changes in plasma potassium concentration $([\mathrm{K}])$ and changes in plasma glucose concentration ([glucose]), blood base excess, and plasma volume. Oral glucose and sodium bicarbonate treatments decreased [K] by 25 and $19 \%$, respectively, whereas the combination of both compounds caused an intermediate $[\mathrm{K}]$ decline $(22 \%)$. For the glucose treatment, the decline in $[\mathrm{K}]$ was only associated with changes in plasma [glucose] (partial $\mathrm{R}^{2}$ $=0.19)$. In $\mathrm{NaHCO}_{3}$-treated calves, $[\mathrm{K}]$ decline was associated with change of extracellular volume (partial $\mathrm{R}^{2}=0.31$ ) and blood base excess (partial $\mathrm{R}^{2}=0.19$ ). When glucose and $\mathrm{NaHCO}_{3}$ were combined, $[\mathrm{K}]$ decline was associated with changes in plasma volume (partial
\end{abstract}

Received May 5, 2011.

Accepted July 25, 2011.

${ }^{1}$ Corresponding author: waltergruenberg@yahoo.com
$\left.\mathrm{R}^{2}=0.30\right), \mathrm{BE}\left(\mathrm{R}^{2}=0.22\right)$, and [glucose] (partial $\mathrm{R}^{2}=$ 0.03 ). Our results indicate that glucose lowers plasma $[\mathrm{K}]$ mainly through an insulin-dependent intracellular translocation of $\mathrm{K}$, whereas $\mathrm{NaHCO}_{3}$ causes hypokalemia through hemodilution followed by intracellular translocation of $\mathrm{K}$ caused by the strong ion effect. The combination of glucose and $\mathrm{NaHCO}_{3}$ at the dosage used in this study does not have an additive hypokalemic effect. When combined, hemodilution and strong ion effect have the strongest effect on plasma $[\mathrm{K}]$, whereas the insulin-dependent effect of glucose appears to be blunted.

Key words: calf, potassium, glucose, sodium bicarbonate

\section{INTRODUCTION}

Hyperkalemia is a common complication in calves with severe neonatal diarrhea and has the potential to cause life-threatening bradyarrhythmias. The presumed underlying mechanisms are decreased renal perfusion in dehydrated calves and decreased intracellular translocation of potassium (K) in states of acidemia. Although treatment with glucose or insulin is well established for the treatment of hyperkalemia, the efficacy of sodium bicarbonate is controversial in several species (Kim and Han, 2002; Weisberg, 2008; Youn and McDonough, 2009). In general, hyperkalemia in diarrheic calves is not addressed as an individual problem because correction of the $\mathrm{K}$ disequilibrium presumably occurs in response to the administration of oral and parenteral glucose, electrolytes, and sodium bicarbonate together with large amounts of fluids, which is common practice in these animals. To our knowledge, the effect and underlying mechanism of sodium bicarbonate and glucose on $\mathrm{K}$ homeostasis in calves has not yet been investigated. Therefore, the objectives of the present experiment were to study the effect of both compounds as well as the combination thereof on plasma K concentration when administered orally to healthy, nonanuric, nonacidotic neonatal calves and to explore the underlying mechanisms. 


\section{MATERIALS AND METHODS}

All methods were approved by the Animal Care and Use Committee, Berlin, Germany (permit \# 0384/10).

\section{Animals, Housing, and Feeding}

Nine healthy male Holstein-Friesian calves were obtained from a commercial dairy farm between $\mathrm{d} 7$ and 14 of life. Calves were kept unrestrained in groups of 2 in straw-bedded stalls in a climate-controlled environment. All animals were allowed a 6- to 8-d acclimation period during which their health status was monitored. A commercial casein-based milk replacer (Sprayfo Sprint, Sloten, Diepholz, Germany) with $22.5 \%$ CP, $16 \%$ crude fat, $0.1 \%$ crude fiber, and $7.0 \%$ crude ash was fed twice daily (150 g of milk replacer/L of water, $60 \mathrm{~mL}$ of solution $/ \mathrm{kg}$ of $\mathrm{BW}, \mathrm{pH}=6.5$, temperature $=38^{\circ} \mathrm{C}$ ) at standardized times from a nipple bucket. Calves had access to fresh water ad libitum except for during an 8-h period following each experimental treatment. All calves underwent a complete physical exam at least $12 \mathrm{~h}$ before the first treatment and had jugular catheters and urine collection bags fitted, as follows. A 16-gauge, 83-mm catheter (Angiocath, Becton Dickinson, Heidelberg, Germany) was aseptically fitted in a jugular vein, and a urine collection bag with a connection port to attach tubing for urine collection was glued onto the hair around the preputium of the calves. Catheters and urine collection bags remained in place for $5 \mathrm{~d}$ until the end of the study. Venous catheterization was performed after sedation with xylazine $(0.2 \mathrm{mg} / \mathrm{kg}$ of BW i.m.), aseptic preparation of the skin, and injection of $1 \mathrm{~mL}$ of $4 \%$ procaine into and under the skin over the jugular vein to be catheterized. A small stab incision through the skin was made with a scalpel to facilitate the catheter placement. The catheter was placed into the vein, connected to an extension set (Discofix C-3, $10 \mathrm{~cm}$, Braun Melsungen AG, Melsungen, Germany), and secured in place with suture material. Catheters were flushed every 8 to $12 \mathrm{~h}$ with heparinized $0.9 \%$ $\mathrm{NaCl}$ solution (40 $\mathrm{U}$ of heparin $/ \mathrm{mL}$ ).

\section{Experimental Protocol}

Studies were conducted between d 15 and 24 of age and at least $12 \mathrm{~h}$ after the last feeding. Calves on trial were restrained with a fence placed in the stall in such manner that they could sit and stand easily but could not turn in a complete circle.

Treatment order was assigned randomly for each individual animal with a washout period of $48 \mathrm{~h}$ between treatments. On each trial day, calves were tube-fed between 0800 and $0815 \mathrm{~h}$ with one of following test solutions at approximately $38^{\circ} \mathrm{C}: 25.2 \mathrm{~g}$ of $\mathrm{NaHCO}_{3}$ in 2
$\mathrm{L}$ of tap water (NaBic, $150 \mathrm{mmol} / \mathrm{L}$ ); $118 \mathrm{~g}$ of glucose monohydrate in $2 \mathrm{~L}$ of tap water (Gluc, $300 \mathrm{mmol} / \mathrm{L}$ ), or $25.2 \mathrm{~g}$ of $\mathrm{NaHCO}_{3}$ plus $118 \mathrm{~g}$ of glucose in $2 \mathrm{~L}$ of tap water (Combi). The solutions of groups NaBic and Gluc were formulated to be isotonic $(300 \mathrm{mOsm} / \mathrm{L})$. The test solution of group Combi was of double tonicity $(600 \mathrm{mOsmol} / \mathrm{L})$ but formulated to match the Na:glucose ratio of 1:2 on a molar basis recommended for oral electrolyte solutions for calves (Smith, 2009). Tube feeding was chosen over voluntary intake to standardize the ingestion time of the test solution, because different palatabilities of salt and glucose solution were likely to affect ingestion time.

Venous blood samples were obtained from the jugular catheter at $0 \mathrm{~min}$ (immediately before tube feeding) as well as 30,60, 90, 120, 150, 180, 210, 240, 300, 360,420 , and 480 min after tube feeding. Blood for blood gas analysis was collected anaerobically into heparinized 1-mL plastic syringes that were immediately capped and analyzed within 10 min of collection. Blood for biochemical analysis was collected into 10$\mathrm{mL}$ tubes containing lithium heparin as anticoagulant and centrifuged within $10 \mathrm{~min}$ at 1,500 $\times g$ for $15 \mathrm{~min}$ at room temperature. Plasma was harvested and stored at $-24^{\circ} \mathrm{C}$ until analyzed. Immediately after administration of the test solution, tubing connected to a $3-\mathrm{L}$ collection vial was attached to the connecting port of the urine collection bag to allow volumetric urine collection for a period of $8 \mathrm{~h}$ after the start of treatment. At the end of the 8-h period, the volume of urine collected was determined. A urine sample was obtained after thorough mixing and immediately frozen and stored at $-24^{\circ} \mathrm{C}$ until analyzed.

Plasma samples were assayed for the concentrations of sodium ([Na], flame atomic absorption spectrometry), potassium $([\mathrm{K}]$, flame atomic absorption spectrometry), chloride ([Cl], ion selective electrode), glucose ([gluc], hexokinase method), and total protein ([TP $]$, biuret method). An automated chemistry analyzer (Cobas Mira Plus, Roche, Basel, Switzerland) was used for the plasma biochemical analysis, and flame atomic absorption spectrometry was conducted on a Solaar M6 spectrometer (Thermo Fisher Scientific, Schwerte, Germany). Blood gas analysis was performed on an automated blood gas analyzer (Radiometer AVL5, Brønshøj, Denmark). Measured values for blood $\mathrm{pH}$, partial pressure of $\mathrm{CO}_{2}$, and partial pressure of $\mathrm{O}_{2}$ were corrected for rectal temperature and used to calculate the actual $\mathrm{HCO}_{3}$ concentration $\left(\left[\mathrm{HCO}_{3}\right]\right)$ and the actual base excess (BE) using standard equations (Burnett et al., 1995). Urine was analyzed for urine [K] as described for plasma.

To crudely estimate volume changes of extracellular space that might have occurred after treatment, 
changes in plasma volume at each time point $i$ relative to time zero $\left(\mathbf{T}_{\mathbf{0}}\right)$ were extrapolated from the changes in $[\mathrm{TP}]$ as follows: $\operatorname{VolDiff}_{i}(\%)=\left(\left[\mathrm{TP}_{0}\right]-\left[\mathrm{TP}_{i}\right]\right) \times$ $100 /\left[\mathrm{TP}_{i}\right]$ (Nouri and Constable, 2006).

The strong ion difference $\left(\mathbf{S I D}_{3}\right)$ was calculated from the plasma concentrations of $\mathrm{Na}, \mathrm{K}$, and $\mathrm{Cl}$ as follows: $\mathrm{SID}_{3}(\mathrm{mEq} / \mathrm{L})=([\mathrm{Na}]+[\mathrm{K}])-[\mathrm{Cl}]$. To determine the kinetics of glucose absorption the increment of [gluc] relative to $\mathrm{T}_{0}$ was determined for each time point $i$ using the equation GlucDIFF ${ }_{i}=\left[\operatorname{gluc}_{i}\right]-\left[\operatorname{gluc}_{0}\right]$. In a similar manner, the changes (Diff) in concentration for each time point relative to $\mathrm{T}_{0}$ were determined for $\mathrm{K}$ $\left(\mathrm{KDiff}_{i}\right), \mathrm{Na}\left(\mathrm{NaDiff}_{i}\right), \mathrm{BE}\left(\mathrm{BEDiff}_{i}\right)$, and $\mathrm{SID}_{3}$ (SID${ }_{3}$ Diff $_{i}$ ). The change of the plasma $[\mathrm{K}]$ was corrected for plasma volume changes using the equation

$$
\operatorname{VolCorrKDiff}_{i}(\mathrm{mmol} / \mathrm{L})=\operatorname{KDiff}_{i}+\operatorname{VolDiff}_{i}{ }
$$

The maximum decrement of $[\mathrm{K}]$ relative to $\mathrm{T}_{0}\left(\mathbf{C}_{\text {min- }}\right.$ Kdiff; baseline $[\mathrm{K}]$ - lowest $[\mathrm{K}]$ measured in the first 120 min) as well as the time at which the lowest $[\mathrm{K}]$ was measured ( $\mathbf{T}_{\text {minKdiff }}$ ) were obtained from a plot of the plasma concentration-time data.

The area under the plasma [gluc], $\left[\mathrm{HCO}_{3}\right], \mathrm{BE}$, and VolDiff increment time curve for the first $4 \mathrm{~h}$ after treatment $\left(\mathrm{AUC}_{\mathrm{GlucDiff-240}}, \mathbf{A U C}_{\mathrm{HCO3Diff-240}}, \mathbf{A U C}_{\mathrm{BE}-}\right.$ Diff-240) as well as the area under the plasma $[\mathrm{K}]$ decrement-time curve for the same time interval $\left(\mathbf{A} \mathbf{U C}_{\mathbf{K}}\right.$ diff-240) were calculated using the trapezoidal rule.

\section{Statistical Analysis}

Data are expressed as mean \pm standard deviation (SD) or as median and interquartile range. A $P$ value $<0.05$ was considered significant. Values were log-transformed when necessary to achieve a normal distribution. Repeated-measures ANOVA was used to detect differences in measured parameters between treatment groups and over time using PROC MIXED (SAS 9.2, SAS Institute Inc., Cary, NC). Bonferroniadjusted $P$-values were used to assess differences within and between treatment groups whenever the $F$-test was significant.

The association between several determined parameters and KDiff ${ }_{i}$ was tested using forward and backward multivariate stepwise linear regression analysis $(P<$ 0.05 for entry and exit) only including values obtained within the first $120 \mathrm{~min}$ of treatment. This time interval was chosen because the decline in plasma $[\mathrm{K}]$ occurred and reached its nadir in all groups within this period. The analysis used a dummy variable coding for each calf, which accounted for between-calf variability, thereby increasing the precision with which slope and intercept coefficients for the regression line can be es- timated (Glantz and Slinker 1990). This analytical approach enforces a uniform slope but different intercept value for each calf; this approach is appropriate whenever the slopes are similar, as in this study. Dummy variables $\left(\mathrm{C}_{1}\right.$ to $\left.\mathrm{C}_{\mathrm{n}}\right)$ were defined as follows: $\mathrm{C}_{1}=1$ if calf $i(i<n),-1$ if calf $=n$, and 0 otherwise. The parameters included in the multivariate stepwise linear regression analysis were VolDiff, as a proxy for changes in plasma and thus extracellular volume; GlucDiff, as a proxy for the increment of the plasma insulin concentration following intestinal glucose absorption; BEDiff and $\mathrm{SID}_{3}$ Diff as a proxy for change of the strong ion difference, according to Stewart's strong ion model; and $\mathrm{HCO}_{3}$ Diff as a proxy for the change in acid-base homeostasis, according to the Henderson-Hasselbalch model. Furthermore, NaDiff was included to identify a possible Na-dependent effect on plasma $[\mathrm{K}]$. Pearson correlation analysis stratified by treatment group was conducted to identify possible associations of the total renal $\mathrm{K}$ excretion with the $\mathrm{AUC}_{\mathrm{KDiff}-240}$ and $\mathrm{C}_{\text {min-KDiff }}$ in plasma.

The SAS statistical software package was used for analysis (SAS 9.2; SAS Institute Inc.).

\section{RESULTS}

The plasma $[\mathrm{K}]$-time curve declined with a similar slope and duration in all 3 treatment groups (Figure 1, upper panel). Although neither a treatment effect nor a treatment $\times$ time interaction was identified, the time effect on plasma $[\mathrm{K}]$ was significant $(P<0.0001)$, with a decline within 30 min of oral treatment in all groups. The nadir of the plasma $[\mathrm{K}]$ decrement $\left(\mathrm{T}_{\min -\mathrm{KDiff}}\right)$ was reached after $87 \pm 32 \mathrm{~min}$ in group NaBic, $110 \pm 56$ min in group Gluc, and $124 \pm 54$ min in group Combi, with maximum decrements of plasma $[\mathrm{K}]\left(\mathrm{C}_{\text {min-KDiff }}\right)$ of $-1.2 \pm 0.2,-0.8 \pm 0.4$, and $-1.0 \pm 0.4 \mathrm{mmol} / \mathrm{L}$ in groups Gluc, NaBic, and Combi, respectively. These declines were equivalent to 25,19 , and $22 \%$ of the baseline plasma $[\mathrm{K}]$ concentrations in groups Gluc, NaBic, and Combi, respectively. Treatment effects were not significant for either $\mathrm{T}_{\min -K D i f f}$ or $\mathrm{C}_{\text {min-KDiff. Plasma }}[\mathrm{K}]$ remained below baseline values for at least $240 \mathrm{~min}$ in all groups (Figure 1a). The analysis of the plasma KDiff-time data revealed a significant time effect but also a treatment effect $(P=0.0227$, Figure $1 \mathrm{~b})$, with a significantly more pronounced decrement in plasma $[\mathrm{K}]$ in Gluc compared with NaBic $(P=0.0009)$. The area under the $[\mathrm{K}]$ decrement time curve $\left(\mathrm{AUC}_{\mathrm{KDiff}-240}\right)$ was $-182 \pm 49 \mathrm{mmol} / \mathrm{L} \cdot \mathrm{min}$ in Gluc, $-119 \pm 70$ $\mathrm{mmol} / \mathrm{L} \cdot \mathrm{min}$ in NaBic, and $-162 \pm 76 \mathrm{mmol} / \mathrm{L} \cdot \mathrm{min}$ in Combi. The treatment effect on $\mathrm{AUC}_{\mathrm{KD} \text { iff-240 }}$ was not significant. The analysis of the volume-corrected decrement in plasma $[\mathrm{K}]$ (Figure 1c) yielded significant time 


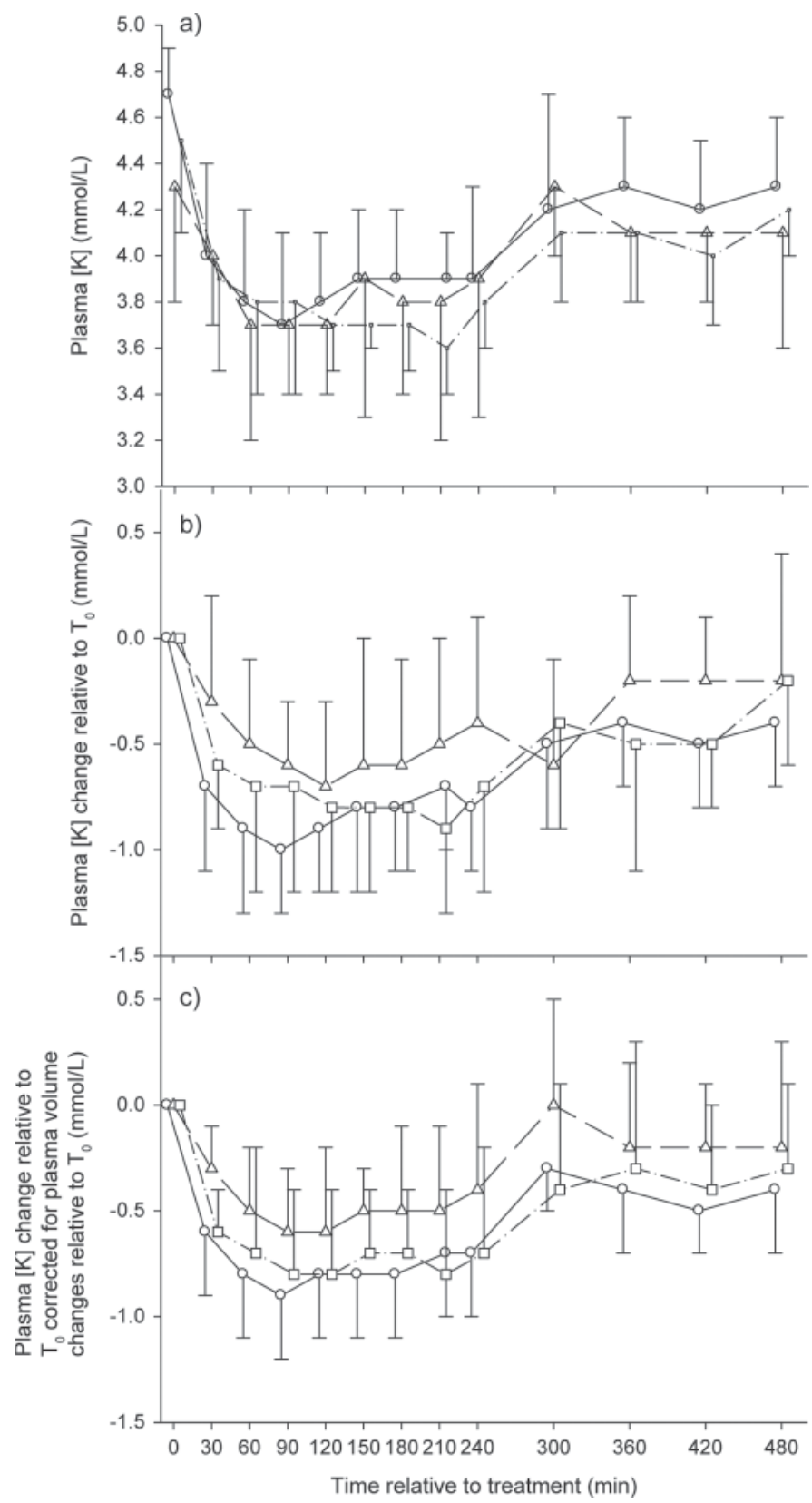

Figure 1. Mean plasma $[\mathrm{K}] \pm \mathrm{SD}$ (upper panel), mean change in plasma $[\mathrm{K}]$ relative to time zero $\left(\mathrm{T}_{0}\right) \pm \mathrm{SD}$ (middle panel), and mean change in plasma $[\mathrm{K}]$ relative to $\mathrm{T}_{0} \pm \mathrm{SD}$ corrected for changes in plasma volume relative to $\mathrm{T}_{0}$ (lower panel) in groups Gluc $(300 \mathrm{mmol} / \mathrm{L}$ of glucose; O), NaBic $\left(150 \mathrm{mmol} / \mathrm{L} \mathrm{NaHCO}_{3} ; \Delta\right)$, and Combi $(150$ $\mathrm{mmol} / \mathrm{L} \mathrm{NaHCO}{ }_{3}+300 \mathrm{mmol} / \mathrm{L}$ glucose; $\square$ ). Samples were obtained at the same time points in all groups but time points were spread apart slightly to improve clarity.

and treatment effects $(P=0.0185)$, with a significantly smaller decline of the volume-corrected plasma $[\mathrm{K}]$ in NaBic compared with Gluc $(P=0.0077)$ and Combi $(P=0.0347)$.

Renal K excretion did not differ significantly between groups during the $8 \mathrm{~h}$ following treatment (Figure 2).

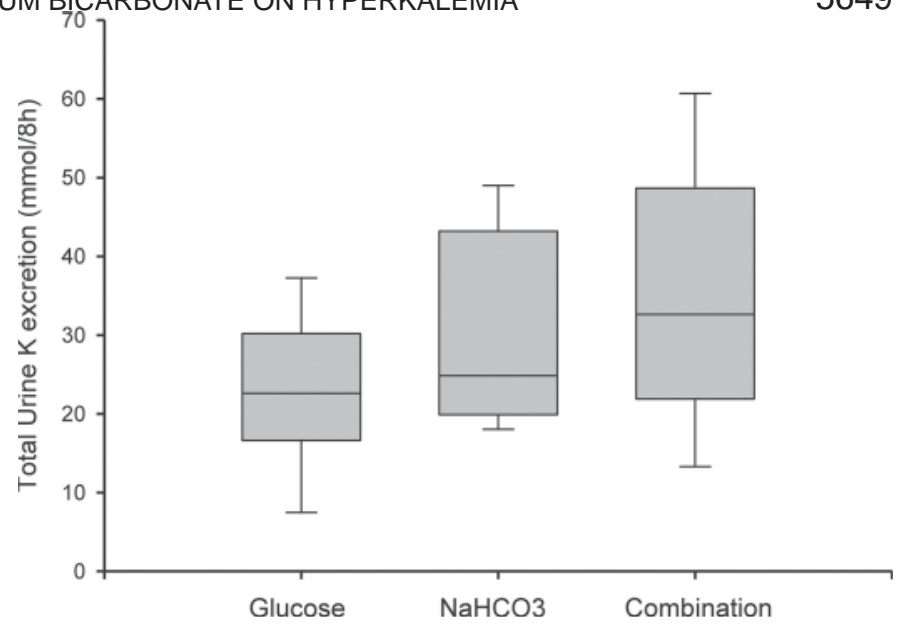

Figure 2. Box and whisker plots representing total renal K excretion during $8 \mathrm{~h}$ following treatment stratified by group. Box and whisker plots represent median (horizontal line), lower and upper quartile (bottom and top of box, respectively), and second and ninth percentiles (lower and upper end of whiskers, respectively). Group differences were not significant.

Pearson correlation analysis stratified by group revealed significant associations of the total renal $\mathrm{K}$ excretion with $\mathrm{C}_{\text {min-Kdiff }}(\mathrm{R}=0.861 ; P=0.0029)$ and with $\operatorname{AUC}_{\mathrm{KDiff-240}}(\mathrm{R}=0.764 ; P=0.0164)$ in Gluc only, indicating that, in this group, the more pronounced the decline in plasma $[\mathrm{K}]$, the greater the decrease in total amount of $\mathrm{K}$ excreted in urine. Urine volumes produced over the $8 \mathrm{~h}$ immediately following oral treatment were (median and interquartile range) $1,500 \mathrm{~mL}$ $(1,150-1,700 \mathrm{~mL}), 1,170 \mathrm{~mL}(1,150-1,950 \mathrm{~mL})$, and $980 \mathrm{~mL}(750-2,000 \mathrm{~mL})$ in Gluc, NaBic, and Combi, respectively.

Slope and amplitude of the plasma [glucose]-time curve were similar in Gluc and Combi (Figure 3). Group differences for $\mathrm{C}_{\text {max-GlucDiff, }}, \mathrm{T}_{\text {max-GlucDiff, }}$ and $\mathrm{AUC}_{\mathrm{GlucDiff} 240}$ were identified between NaBic (not having received any glucose) and the other groups. No differences between Gluc and Combi were found.

Plasma volume changes extrapolated from the change in plasma $[\mathrm{TP}]$ are presented in Figure 4. Analysis of variance revealed a significant time effect $(P=0.0321)$ but not a treatment or treatment $\times$ time interaction. Plasma volume was significantly increased from 60 to 150 min posttreatment. No treatment effect was determined on $\mathrm{AUC}_{\text {VolDiff-240. }}$.

Changes in venous blood $\mathrm{pH}$ and $\mathrm{BE}$ over time are presented in Figure 5. Although venous blood $\mathrm{pH}$ was, and remained, within normal limits throughout the study in all groups, numerical increases after oral treatment were observed in NaBic and Combi. Venous blood $\mathrm{pH}$ changes over time were significant in NaBic only. The blood BE increased significantly above baseline 
values within 60 min of treatment in groups NaBic and Combi and remained above baseline values until the end of the study period. No time effect was observed in Gluc, this group not having received any sodium bicarbonate (Figure 5). Accordingly, the area under the BEDiff-time curve was significantly smaller for Gluc compared with the other 2 groups, whereas the difference between $\mathrm{NaBic}$ and Combi was not significant.

Forward multiple stepwise regression analysis conducted by group with KDiff as dependent variable and VolDiff, BEDiff, $\mathrm{HCO}_{3}$ Diff, $\mathrm{SID}_{3}$ Diff, GlucDiff, and NaDiff as independent variables yielded a final model in which GlucDiff remained as the sole significant independent variable (partial $\mathrm{R}^{2}=0.193, P=0.0058$ ) in Gluc. In NaBic, VolDiff (partial $\mathrm{R}^{2}=0.3087, P<$ 0.0001 ) and BEDiff (partial $\mathrm{R}^{2}=0.1864, P<0.0001$ ) remained in the final model, and in Combi, VolDiff (partial $\left.\mathrm{R}^{2}=0.3017, P<0.0001\right)$, BEDiff (partial $\mathrm{R}^{2}=$ $0.2197, P<0.0001$ ), and GlucDiff (partial $\mathrm{R}^{2}=0.0319$, $P=0.0287)$ remained in the final model.

\section{DISCUSSION}

The results presented here revealed a marked plasma potassium-lowering effect of similar extent of glucose
(600 mmol) and sodium bicarbonate $(300 \mathrm{mmol})$ when administered orally to healthy neonatal calves. The combination of both substances in one treatment did not result in a noticeable additive effect on extracellular K homeostasis.

Several mechanisms with the potential to cause a marked decline in plasma $[\mathrm{K}]$ must be considered. In addition to increased renal $\mathrm{K}$ excretion and altered transcellular distribution of $\mathrm{K}$, an osmotically driven volume expansion of the extracellular space is also likely to reduce plasma $[\mathrm{K}]$. Our current understanding of $\mathrm{K}$ homeostasis in mammals suggests that rapid correction of postprandial increases in plasma $[\mathrm{K}]$, having the potential to jeopardize the equilibrium of extracellular $[\mathrm{K}]$, is achieved mainly through intracellular shifts of $\mathrm{K}$ (Youn and McDonough, 2009). In contrast, renal $\mathrm{K}$ regulation, although very efficient, has a lag time of hours and thus is unlikely to be responsible for the changes in plasma $[\mathrm{K}]$ observed within 60 to $120 \mathrm{~min}$ of treatment (Youn and McDonough, 2009). Similarly, the aldosterone-dependent $\mathrm{K}$ absorption from the large intestine lags several hours behind feed intake, because ingesta must reach the lower intestinal tract; thus, this was not considered in the present study (Rose and Post, 2001).

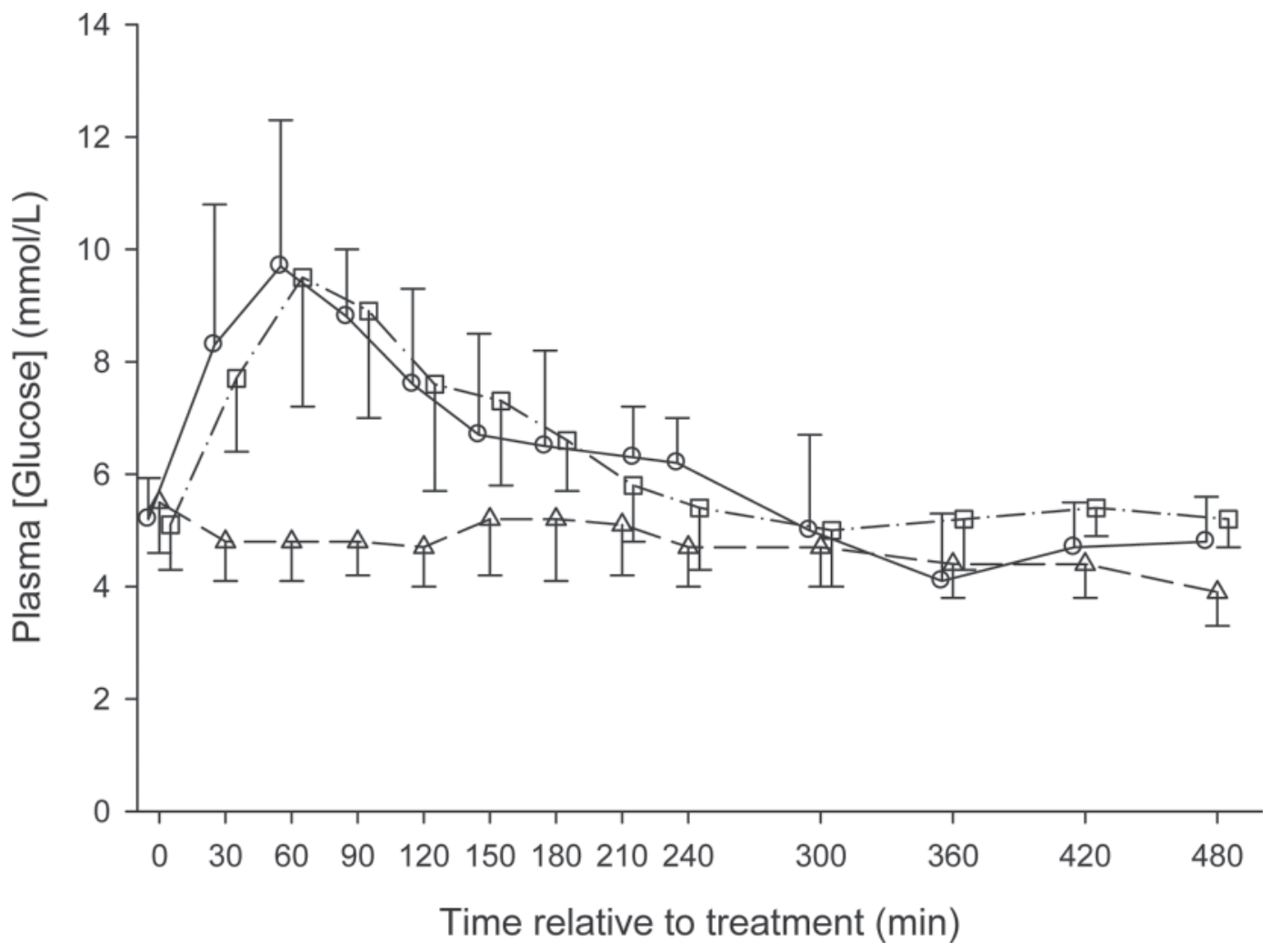

Figure 3. Mean plasma [glucose] $\pm \mathrm{SD}$ relative to treatment in groups Gluc $(300 \mathrm{mmol} / \mathrm{L}$ of glucose; $\bigcirc), \mathrm{NaBic}\left(150 \mathrm{mmol} / \mathrm{L} \mathrm{NaHCO} ;{ }_{3}\right)$, and Combi $\left(150 \mathrm{mmol} / \mathrm{L} \mathrm{NaHCO}_{3}+300 \mathrm{mmol} / \mathrm{L}\right.$ glucose; $\left.\square\right)$. Samples were obtained at the same time points in all groups but time points were spread apart slightly to improve clarity. 


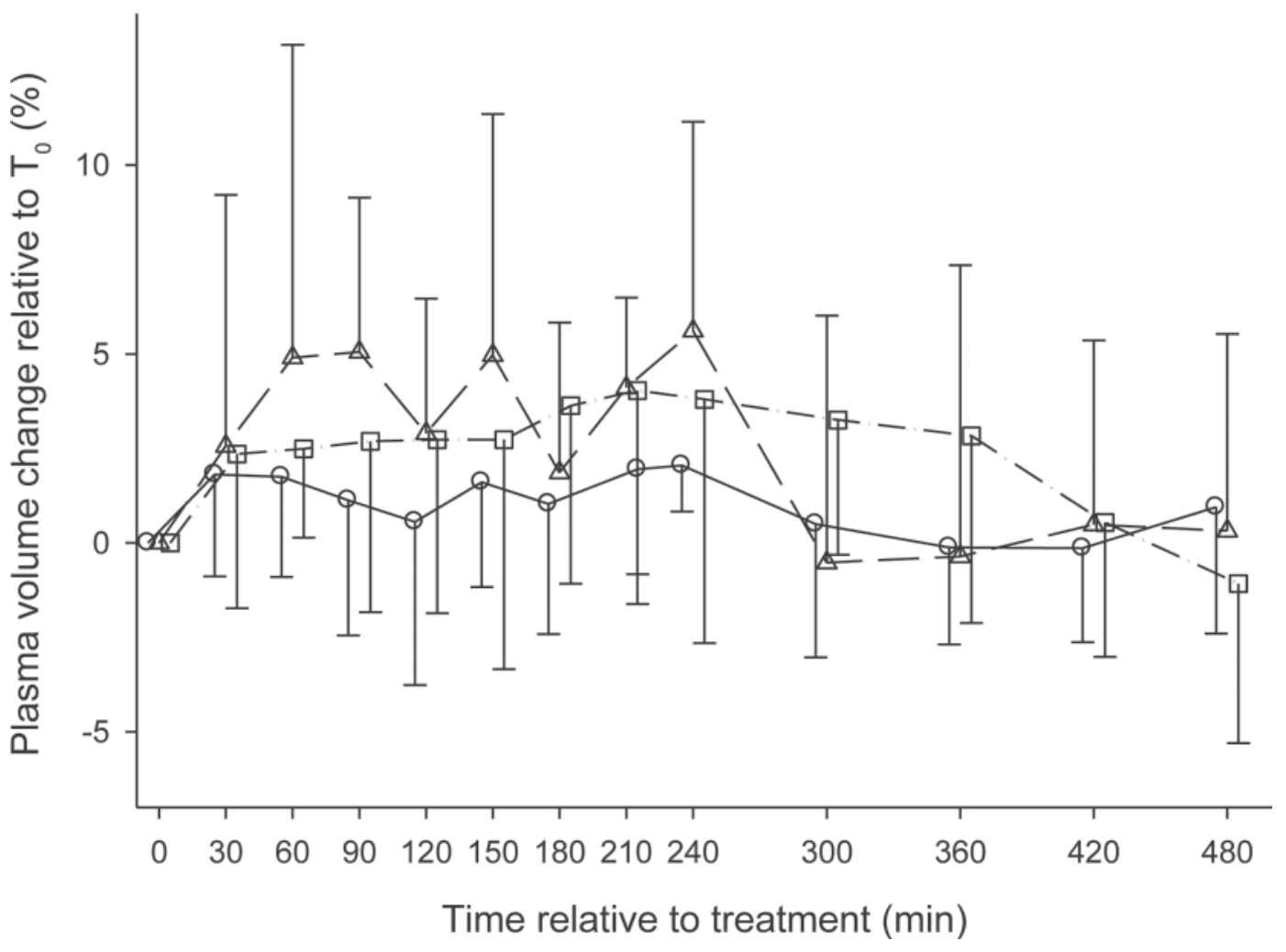

Figure 4. Mean plasma volume changes $\pm \mathrm{SD}$ relative to treatment in groups Gluc (300 mmol/L of glucose; $\mathrm{O}$ ), NaBic (150 mmol/L $\left.\mathrm{NaHCO}_{3} ; \Delta\right)$, and Combi $\left(150 \mathrm{mmol} / \mathrm{L} \mathrm{NaHCO}_{3}+300 \mathrm{mmol} / \mathrm{L}\right.$ glucose; $\left.\square\right)$. Samples were obtained at the same time points in all groups but time points were spread apart slightly to improve clarity.

Compartmental shifts of $\mathrm{K}$ have been reported not only after glucose administration, but also after administration of alkalinizing agents such as sodium bicarbonate, and are associated with marked declines of extracellular [K] (Burnell et al., 1956; Burnell and Scribner, 1957; Smith and Etteldorf, 1961; Hundal et al., 1992). Alteration of the transcellular distribution of $\mathrm{K}$ triggered by insulin and sodium bicarbonate is thought to occur through different pathways. Insulin-dependent cellular K uptake has been proposed to be mediated through activation of the Na-K-ATPase in response to an elevated plasma insulin concentration (Hundal et al., 1992). In contrast, alkalinization with sodium bicarbonate was proposed to cause a decrease in the extracellular $\mathrm{H}^{+}$concentration, resulting in increased $\mathrm{H}^{+}$transport out of the cell in exchange for $\mathrm{Na}$ through the $\mathrm{Na} / \mathrm{H}$ exchanger, making extra $\mathrm{Na}$ available to be exchanged for $\mathrm{K}$ through the Na-K-ATPase pump.

Plasma volume expansion must be considered whenever osmotically active compounds readily absorbed from the gastrointestinal tract such as glucose or sodium bicarbonate are administered orally. The positive effect on plasma volume expansion of oral electrolyte solutions containing large amounts of glucose in calves has been reported previously (Brooks et al., 1996). In humans, plasma volume expansion was discussed as a probable factor contributing to the mild plasma potassium-lowering effect in some patients treated intravenously with sodium bicarbonate (Blumberg et al., 1992). Comparison of the crude and volume-corrected changes of plasma $[\mathrm{K}]$ in the current study suggests that plasma volume expansion had the strongest effect on the decline of plasma $[\mathrm{K}]$ in NaBic and to some extent in Combi, but had no noticeable effect in Gluc (Figure 1).

Although the different test solutions used in the present study could have altered intestinal $\mathrm{K}$ absorption, this effect was considered unlikely to affect the results presented here because none of the test solutions contained K. Therefore, intestinal $\mathrm{K}$ absorption in the proximal intestinal tract was considered to be equally low in all groups.

Although baseline plasma $[\mathrm{K}]$ did not differ significantly between groups, numerical differences between groups were larger than could be considered negligible (Figure 1). It remains unclear to what extent these differences in baseline concentrations might have contributed to the observed treatment effects. 


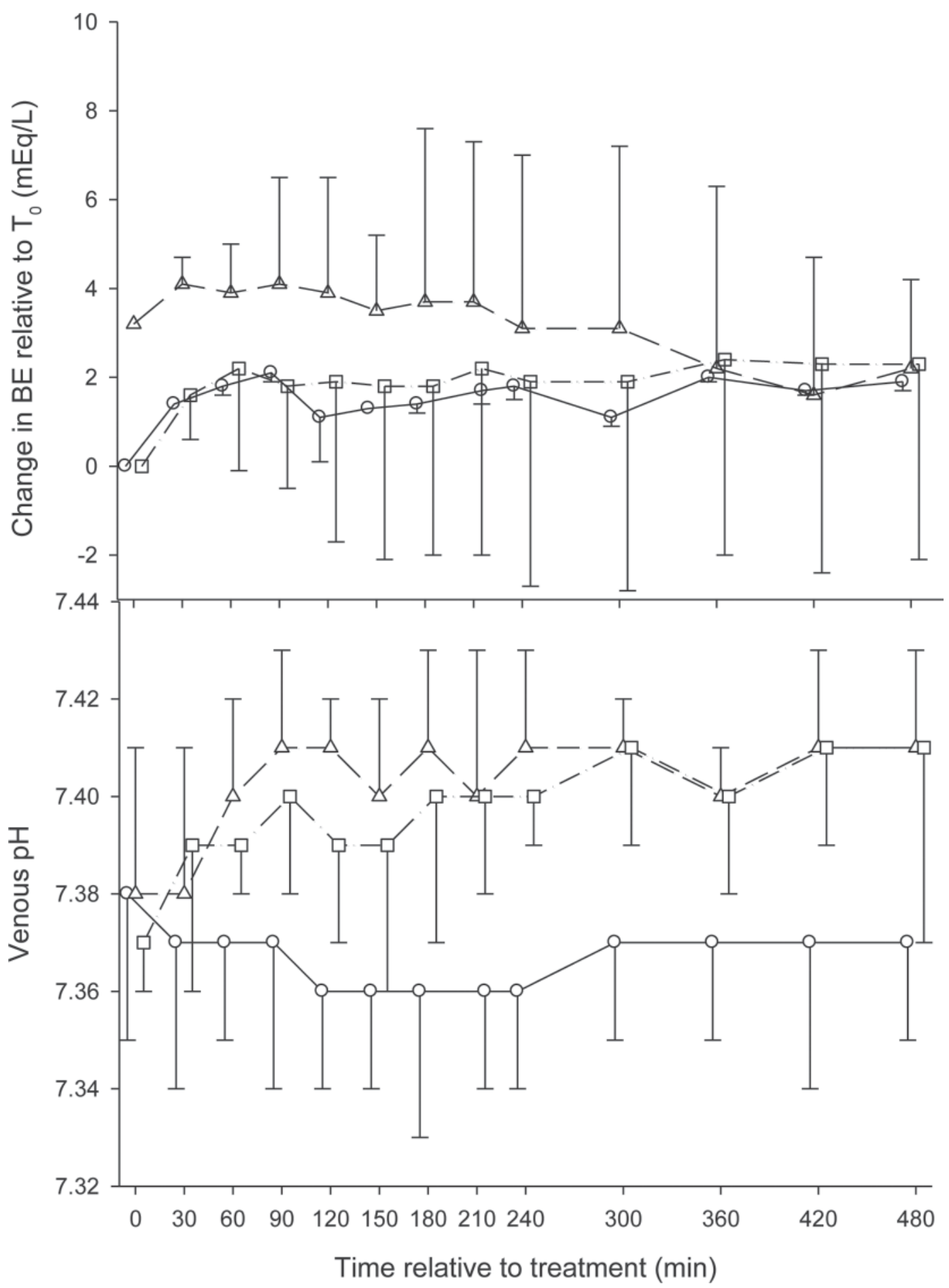

Figure 5. Mean change in blood base excess (BE) relative to time zero $\left(\mathrm{T}_{0}\right) \pm \mathrm{SD}$ (upper panel) and mean venous blood $\mathrm{pH} \pm \mathrm{SD}$ (lower panel) in groups Gluc $\left(300 \mathrm{mmol} / \mathrm{L}\right.$ of glucose; ), NaBic $(150 \mathrm{mmol} / \mathrm{L} \mathrm{NaHCO} ; \quad \Delta)$, and Combi $\left(150 \mathrm{mmol} / \mathrm{L} \mathrm{NaHCO}_{3}+300 \mathrm{mmol} / \mathrm{L}\right.$ glucose; $\square)$. Samples were obtained at the same time points in all groups but time points were spread apart slightly to improve clarity.

In the present study, proxies were used to assess the quantitative contribution of some of the above-mentioned mechanisms to the observed decline in plasma [K] during the first 120 min following oral treatment.
Specifically, the changes in plasma glucose concentration determined during the first 120 min after treatment were used as a crude proxy for the concomitant increase of plasma insulin concentrations to assess the effect of 
insulin-dependent cellular $\mathrm{K}$ uptake. This approach is supported by findings in monogastric species and calves indicating that the increase of plasma insulin concentration after oral carbohydrate administration parallels the increase of plasma glucose (Stanley et al., 2002; Muto et al., 2005).

Although total renal $\mathrm{K}$ excretion did not differ between groups following oral treatment, the strong correlation of the maximum decrement in plasma $[\mathrm{K}]$ $\left(\mathrm{C}_{\text {min-Kdiff }}\right)$ and $\mathrm{AUC}_{\mathrm{KDiff}-240}$ with the total amount of $\mathrm{K}$ excreted in urine during $8 \mathrm{~h}$ following treatment suggest the presence of a treatment effect in calves treated with oral glucose. This analysis does not allow us to determine the chronology of events, but the rapid onset of the intracellular translocation of $\mathrm{K}$ and the lag time of several hours of renal $\mathrm{K}$ regulation make it probable that decreased renal $\mathrm{K}$ excretion is a consequence of decreased availability of extracellular $\mathrm{K}$ in animals treated with glucose. In contrast, in groups $\mathrm{NaBic}$ and Combi, where the decline in plasma $[\mathrm{K}]$ was numerically smaller and plasma volume expansion was determined to be the main factor responsible for the decrease in plasma $[\mathrm{K}]$, the total amount of $\mathrm{K}$ available in the extracellular space and thus filtered in the renal glomeruli would be altered to a lesser degree.

The regression analysis conducted to assess the relative importance of each of the considered mechanisms for the observed plasma potassium-lowering effect indicated that in calves treated with oral glucose alone, hypokalemia was triggered mainly by the insulin-dependent intracellular shift of $\mathrm{K}$, whereas plasma volume expansion did not appear to contribute to this effect in a significant manner. Oral administration of sodium bicarbonate, in contrast, triggered a decline in plasma $[\mathrm{K}]$ mainly through plasma volume expansion and to a lesser extent through compartmental $\mathrm{K}$ shifts related to the strong ion effect, as determined from the significant association of the decline in plasma $[\mathrm{K}]$ with plasma volume changes and blood BE. When combining both compounds in one treatment, the K-lowering effect was not potentiated and the regression analysis revealed that, in this group, mechanisms driving the decline of plasma $[\mathrm{K}]$ were almost identical to what was observed in animals treated with sodium bicarbonate alone, as suggested by the similar partial $\mathrm{R}^{2}$ of plasma volume change and BE in both groups. In particular, the double osmolarity of the test solution containing glucose and sodium bicarbonate did not enhance plasma volume expansion compared with the treatment with sodium bicarbonate alone. In contrast, the insulin-dependent intracellular $\mathrm{K}$ uptake appeared to have been markedly blunted when glucose was combined with sodium bicarbonate compared with the treatment with glucose alone.

Although our results are in agreement with earlier studies reporting a strong hypokalemic effect of sodium bicarbonate (Burnell et al., 1956; Burnell and Scribner, 1957; Smith and Etteldorf, 1961), recent studies have failed to reproduce any effect of sodium bicarbonate on extracellular $\mathrm{K}$ homeostasis in healthy individuals or in patients with end-stage renal failure (Blumberg et al., 1988; Allon and Shanklin, 1996; Kaplan et al., 1997). The discrepancy between findings presented in this study and reports mentioned above could be related to differences in the dosage of sodium bicarbonate. Dosages used in the trials mentioned above ranged between 2 and $3 \mathrm{mmol} / \mathrm{kg}$ of $\mathrm{BW}$ administered intravenously as a hypertonic solution in between 5 and 60 min (Blumberg et al., 1988; Kaplan et al., 1997). In contrast, the dosage of $300 \mathrm{mmol}$ used in this study for a $40-\mathrm{kg}$ calf is equivalent to approximately $7.5 \mathrm{mmol} /$ $\mathrm{kg}$ of BW, which, even when given orally with rapid intestinal absorption, could have a more pronounced osmotic and alkalinizing effect than the lower dose of sodium bicarbonate administered intravenously.

The role of alkalinization for the hypokalemic effect of sodium bicarbonate is controversial. Whereas some authors proposed that the hypokalemic effect depends on the concomitant presence of acidemia (Alfonzo et al., 2006; Parham et al., 2006), this is disputed by others (Fraley and Adler, 1977). In humans and dogs, even mild acidemia was found to hamper insulin sensitivity of liver and peripheral tissues (Mackler et al., 1951; DeFronzo and Beckles, 1979; Van Putten et al., 1985; Arsenis and Livingston, 1986). Correcting acidemia is therefore believed to enhance insulin sensitivity and thereby the translocation of $\mathrm{K}$ into the cells. Accordingly, in humans, the combination of parenteral glucose and insulin treatment with sodium bicarbonate was found to enhance the hypokalemic effect of insulin (Kim, 1996). The implication of this hypothesis, which is supported by studies (Koch and Kaske, 2008; Coskun et al., 2010) on $\mathrm{K}$ homeostasis in calves would be that the effect of both glucose and sodium bicarbonate, as well as the combination, could have a more pronounced K-lowering effect in diarrheic calves suffering from marked concomitant acidemia. Calves in the present study were healthy and did not have acidemia or metabolic acidosis before or after treatment. Only mild and transient metabolic alkalosis was observed in animals in groups NaBic and Combi, and the combination of glucose with sodium bicarbonate appeared to hamper the effect of glucose on extracellular $\mathrm{K}$ equilibrium rather than enhancing it. 
A possible explanation for this effect is the K-specific insulin resistance that presents an entity different and independent of the glucose-related insulin resistance, which has been described in states of $\mathrm{K}$ deprivation (Nørgaard et al., 1981). Even short-term K deprivation can result in a decrease in insulin-dependent cellular $\mathrm{K}$ uptake of up to $80 \%$, whereas insulin-dependent glucose uptake remains unaffected (Choi et al., 2001). This mechanism has been understood as a protection against postprandial hypokalemia when diets rich in carbohydrates but low in potassium are ingested, as was the case in groups Gluc and Combi in the present study.

The main objective of the present study was to elucidate the mechanism by which glucose and sodium bicarbonate after oral administration alter extracellular $\mathrm{K}$ homeostasis rather than directly comparing the hypokalemic effect of both compounds, as this would be of little value. Even though the same amount of both compounds on a molar basis was administered orally, it is difficult to estimate the amount of sodium bicarbonate absorbed from the gastrointestinal tract because varying amounts of bicarbonate may have been lost upon entering the acidic abomasum. Furthermore, it is unclear if, and to what extent, sodium bicarbonate might have been absorbed from the juvenile omasum in these calves, as this occurs in adult animals. Differences in the hypokalemic effect between groups could therefore be due to differences in bioavailability after oral treatment of both compounds.

In conclusion, we report a marked plasma potassiumlowering effect of both sodium bicarbonate and glucose when administered orally to healthy neonatal calves. For sodium bicarbonate, the plasma potassium-lowering effect appeared to be largely driven by plasma volume expansion followed by intracellular translocation of $\mathrm{K}$, whereas glucose caused a decline in plasma $[\mathrm{K}]$ mainly through an intracellular shift of $\mathrm{K}$. The combination of both compounds did not have an additive effect on extracellular K homeostasis.

\section{REFERENCES}

Alfonzo, A. V. M., C. Isles, C. Geddes, and C. Deighan. 2006. Potassium disorders - Clinical spectrum and emergency management. Resuscitation 70:10-25.

Allon, M., and N. Shanklin. 1996. Effect of bicarbonate administration on plasma in dialysis patients: Interaction with insulin and albuterol. Am. J. Kidney Dis. 28:508-514.

Arsenis, G., and J. N. Livingston. 1986. Isoproterenol reduces insulin stimulation of hexose uptake by rat adipocytes via a postinsulin binding alteration. Endocrinology 119:50-57.

Blumberg, A., P. Weidmann, and P. Ferrari. 1992. Effect of prolonged bicarbonate administration on plasma potassium in terminal renal failure. Kidney Int. 41:369-374.

Blumberg, A., P. Weidmann, S. Shaw, and M. Gnädinger. 1988. Effect of various therapeutic approaches on plasma potassium and major regulation factors in terminal renal failure. Am. J. Med. 85:507-512.

Brooks, H. W., D. G. White, A. J. Wagstaff, and A. R. Michell. 1996. Evaluation of a nutritive oral rehydration solution for the treatment of calf diarrhea. Br. Vet. J. 152:699-708.

Burnell, J. M., and B. H. Scribner. 1957. Serum potassium concentration as a guide to potassium need. J. Am. Med. Assoc. 164:959 962.

Burnell, J. M., M. F. Villamil, B. T. Uyeno, and B. H. Scribner. 1956. The effect in humans of extracellular $\mathrm{pH}$ change on the relationship between serum potassium concentration and intracellular potassium. J. Clin. Invest. 35:935-939.

Burnett, R. W., A. K. Covington, N. Fogh-Andersen, W. R. Kulpmann, and A. H. J. Maas. 1995. Approved IFCC recommendation on definitions of quantities and conventions related to blood gases and pH. Eur. J. Clin. Chem. Clin. Biochem. 33:399-404.

Choi, C. S., C. B. Thompson, P. K. Leong, A. A. McDonough, and J. H. Youn. 2001. Short term $\mathrm{K}^{+}$deprivation provokes insulin resistance of cellular $\mathrm{K}^{+}$uptake revealed with the $\mathrm{K}^{+}$clamp. Am. J. Physiol. Renal Physiol. 280:F95-F102.

Coskun, A., I. Sen, H. Guzelbektes, M. Ok, K. Turgut, and S. Canikli. 2010. Comparison of the effects of intravenous administration of isotonic and hypertonic sodium bicarbonate solutions on venous acid base status in dehydrated calves with strong ion acidosis. J. Am. Vet. Med. Assoc. 236:1098-1103.

DeFronzo, R. A., and A. D. Beckles. 1979. Glucose intolerance following chronic metabolic acidosis in man. Am. J. Physiol. 236:E328E334

Fraley, D. S., and S. Adler. 1977. Correction of hyperkalemia by bicarbonate despite constant blood pH. Kidney Int. 12:354-360.

Glantz, S. A., and B. K. Slinker. 1990. Primer of Applied Regression and Analysis of Variance. McGraw-Hill Book Co., New York, NY.

Hundal, H. S., A. Marette, Y. Mitsumoto, T. Ramlal, R. Blostein, and A. Kip. 1992. Insulin induces translocation of the $\alpha 2$ and $\beta 1$ subunits of the $\left.\mathrm{Na}^{+} / \mathrm{K}^{+}\right)$-ATPase from intracellular compartments to the plasma membrane in mammalian skeletal muscle. J. Biol. Chem. 267:5040-5043.

Kaplan, J. L., L. E. Braitman, W. C. Dalsey, M. Montomery, and A. Mangione. 1997. Alkalinization is ineffective for severe hyperkalemia in nonnephrectomized dogs. Acad. Emerg. Med. 4:93-99.

Kim, H. J. 1996. Combined effect of bicarbonate and insulin with glucose in acute therapy of hyperkalemia in end-stage renal disease patients. Nephron 72:476-482.

Kim, H. J., and S. W. Han. 2002. Therapeutic approach to hyperkalemia. Nephron 92(Suppl. 1):33-40.

Koch, A., and M. Kaske. 2008. Clinical efficacy of intravenous hypertonic saline solution or hypertonic bicarbonate solution in the treatment of inappetent calves with neonatal diarrhea. J. Vet. Intern. Med. 22:202-211.

MacKler, B., H. Lichtenstein, and G. M. Guest. 1951. Effects of ammonium chloride acidosis on the action of insulin in dogs. Am. J. Physiol. 166:191-198.

Muto, S., K. Sebata, H. Watanabe, F. Shoji, Y. Yamamoto, M. Ohashi, T. Yamada, H. Matsumoto, T. Mukouyama, T. Yonekura, S. Namiki, and E. Kusano. 2005. Effect of oral glucose administration on serum potassium concentration in hemodialysis patients. Am. J. Kidney Dis. 46:697-705.

Nørgaard, A., K. Kjeldsen, and T. Clausen. 1981. Potassium depletion decreases the number of $3 \mathrm{H}$-oubain binding sites and the active Na-K transport in skeletal muscle. Nature 293:739-741.

Nouri, M., and P. D. Constable. 2006. Comparison of two oral electrolyte solutions and route of administration on the abomasal emptying rate of Holstein-Friesian calves. J. Vet. Intern. Med. 20:620-626.

Parham, W. A., A. A. Mehdirad, K. M. Biermann, and C. S. Fredman. 2006. Hyperkalemia revisited. Tex. Heart Inst. J. 33:40-47.

Rose, B. D., and T. W. Post. 2001. Introduction to disorders of potassium balance. Pages 822-836 in Clinical physiology of acid-base and electrolyte disorders. B. D. Rose and T. W. Post, ed. McGrawHill, New York, NY. 
Smith, G. W. 2009. Treatment of calf diarrhea: Oral fluid therapy. Vet. Clin. North Am. Food Anim. Pract. 25:55-72.

Smith, H. L., and J. N. Etteldorf. 1961. Parenteral fluid regimes in the treatment of severe diarrhea in infants. J. Pediatr. 58:1-15.

Stanley, C. C., C. C. Williams, B. F. Jenny, J. M. Fernandez, H. G. Bateman, W. A. Nipper, J. C. Lovejoy, D. T. Gantt, and G. E. Goodier. 2002. Effects of feeding milk replacer once versus twice daily on glucose metabolism in Holstein and Jersey calves. J. Dairy Sci. 85:2335-2343.
Van Putten, J. P., T. Wieringa, and H. M. Krans. 1985. Low pH and ketoacids induce insulin receptor binding and postbinding alterations in cultured 3T3 adipocytes. Diabetes 34:744-750.

Weisberg, L. S. 2008. Management of severe hyperkalemia. Crit. Care Med. 36:3246-3251.

Youn, J. H., and A. A. McDonough. 2009. Recent advances in understanding integrative control of potassium homeostasis. Annu. Rev. Physiol. 71:381-401. 